\title{
Caracterização de plintitas e petroplintitas em solos da Depressão Central do Rio Grande do Sul
}

\author{
Plintites and petroplintites characterization in soils from Central Depression of the Rio Grande \\ do Sul state, Brazil
}

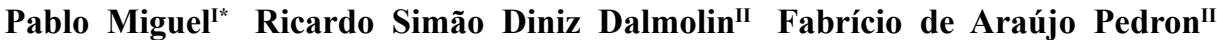 \\ Jessé Rodrigo Fink ${ }^{\mathrm{III}}$ Jean Michel Moura-Bueno ${ }^{\mathrm{I}}$
}

RESUMO

A presença, composição e distribuição de concreções ferruginosas no solo, denominadas de plintitas e petroplintitas, é condicionada às características ambientais da região, atribuindo propriedades intrínsecas ao solo. O objetivo do estudo foi caracterizar plintitas e petroplintitas de solos da Depressão Central do Rio Grande do Sul através da avaliação de características quimicas e mineralógicas dessas feições. Para tanto, procedeu-se à caracterização química, morfológica e granulométrica de três perfis de solo. As concreções ferruginosas presentes em alguns horizontes foram separadas da matriz do solo e submetidas, separadamente, a sucessivos procedimentos de extrações seletivas de $\mathrm{Fe}$ e Al. Os horizontes dos solos apresentaram ampla variação de textura e atributos químicos como pH, S, V, me Matéria Orgânica. Os teores de Feh, Fed e Feo seguiram a tendência: matriz do solo< $<$ plintitas $<$ petroplintitas. Dentre os óxidos de Fe, verificou-se predomínio do mineral hematita em todas as amostras avaliadas.

Palavras-chave: argissolos, plintossolos, plintização, pedogênese, mineralogia.

\section{ABSTRACT}

The presence, composition and distribution of ferruginous concretions in the soil, called plinthite and petroplinthites, are conditioned to the environmental characteristics of the region, attributing intrinsic properties to the soil. The objective of this study was to understand the composition of plinthite and petroplinthites, of soils at the Central Depression of Rio Grande do Sul state, Brazil, by the evaluation of chemical and mineralogical characteristics of these features. For this, chemical, morphological and particle size characterization of three soil profiles, was performed. The ferruginous concretions present in some horizons were separated from the soil matrix and submitted, separately to successive procedures for selective extraction of $\mathrm{Fe}$ and Al. The horizons of the soils showed a wide variation of textures and chemical attributes such as $\mathrm{pH}, \mathrm{S}, V, m$ and MO. The levels of Feh, Feo and Fed followed the trend of the soil matrix < plinthite <petroplinthites. Among the oxides of $\mathrm{Fe}$, there was higher incidence of hematite mineral in all samples.

Key words: ultisols, plintosols, plintization, pedogenesis, mineralogy.

\section{INTRODUÇÃO}

A plintita é uma formação constituída da mistura de argila, pobre em $\mathrm{C}$ orgânico e rica em $\mathrm{Fe}$, ou $\mathrm{Fe}$ e $\mathrm{Al}$, com quartzo e outros materiais. A petroplintita é uma concreção resultante do endurecimento irreversível da plintita (EMBRAPA, 2006). Solos com presença dessas feições possuem uma gênese particular e ocorrem em grandes extensões no Brasil (ANJOS et al., 1995), sendo a sua variabilidade relacionada à hidrologia, litologia, clima e aspectos geomórficos (STOLT et al., 1993).

Segundo ANJOS et al. (2007), a gênese desses materiais ferruginosos está relacionada com a segregação, mobilização, transporte e concentração do ferro no perfil do solo, ocorrendo no terço inferior de vertentes e em planícies aluviais, associadas a períodos de elevada umidade. Na região da Depressão Central do Rio Grande do Sul (RS), plintitas e

IPrograma de Pós-graduação em Ciência do Solo, Universidade Federal de Santa Maria (UFSM), 97105-900, Santa Maria RS, Brasil. E-mail: tchemiguel@yahoo.com.br. Autor para correspondência.

IIDepartamento de Solos, UFSM, RS, Brasil.

IIIDepartamento de Solos, Universidade Federal do Rio Grande do Sul (UFRGS), RS, Brasil. 
petroplintitas ocorrem associados à Plintossolos, Argissolos e Planossolos, principalmente, ocupando posições de transição entre várzeas e o início das coxilhas (STRECK et al., 2008), podendo caracterizar horizontes plíntico, concrecionário ou litoplíntico, conforme EMBRAPA (2006).

A concentração dos materiais ferruginosos pode condicionar a aptidão agrícola do solo devido à formação de camadas semipermeáveis que dificultam a penetração de raízes, movimentação vertical de água no perfil e, em alguns casos, impedimentos à mecanização. Dessa forma, o conhecimento da dinâmica de disposição, formação e evolução dessas feições pode beneficiar o manejo adequado dos solos e contribuir para o entendimento da sua gênese.

O objetivo do estudo foi caracterizar plintitas e petroplintitas de solos da Depressão Central do Rio Grande do Sul através da avaliação de características químicas e mineralógicas dessas feições.

\section{MATERIAL E MÉTODOS}

Foram selecionadas três áreas representativas da Depressão Central do RS, no município de Santa Maria, onde ocorre a presença de plintitas e petroplintitas no solo, sendo descritos três perfis de solo (P1-29 $43^{\circ} \mathrm{S} / 53^{\circ} 43^{\prime} \mathrm{W}, \mathrm{P} 2$ $\left.29^{\circ} 43^{\prime} \mathrm{S} / 53^{\circ} 44^{\prime} \mathrm{W}, \mathrm{P} 3-29^{\circ} 43^{\prime} \mathrm{S} / 53^{\circ} 42^{\prime} \mathrm{W}\right)$, conforme SANTOS et al. (2005), e classificados segundo EMBRAPA (2006). A geologia presente é a Formação Santa Maria, constituída por siltitos argilosos maciços, de cor vermelha, com níveis esbranquiçados de concreções calcárias, subhorizontais de ambiente continental (SARTORI, 2009).

$\mathrm{O}$ regime pluvial anual médio é de $1708 \mathrm{~mm}$, com temperatura média anual de $19,2^{\circ} \mathrm{C}$ (MALUF, 2000). Os três perfis se encontram em condição de terço médio de encosta com altitude de $100 \mathrm{~m}$ e imperfeitamente drenados. O P1 apresenta uso atual com lavoura anual de grãos e o P2 e P3 campo nativo. Foram coletadas amostras de solos de cada horizonte dos perfis selecionados e do material de origem. As amostras que apresentaram plintitas ou petroplintitas foram submetidas à separação manual realizada com o auxílio de artefatos pontiagudos de polietileno, com o solo ainda úmido. Após a separação dos materiais ferruginosos da matriz do solo, todas as amostras foram secas ao ar, peneiradas (malha 2mm) e moídas em almofariz para posterior análise.

Os teores de $\mathrm{Ca}^{2+}, \mathrm{Mg}^{2+}, \mathrm{K}^{+}$foram determinados segundo metodologia preconizada por EMBRAPA (1997). A partir dos dados analíticos, foram calculadas a soma de bases (S), capacidade de troca de cátions $\left(\mathrm{CTC}_{\mathrm{pH}} 7,0\right)$, saturação por bases (V) e saturação por alumínio (m). A determinação de carbono orgânico no solo foi realizada via combustão úmida, pelo método Mébius modificado (NELSON \& SOMMERS, 1996). Para determinação dos teores de ferro $\left(\mathrm{Fe}_{\mathrm{d}}\right)$ e alumínio $\left(\mathrm{Al}_{\mathrm{d}}\right)$ dos óxidos pedogênicos, foram realizadas duas extrações sequenciais com ditionito-citrato-bicarbonato de sódio a $80^{\circ} \mathrm{C}$ (MEHRA \& JACKSON, 1960). O teor de ferro $\left(\mathrm{Fe}_{\mathrm{o}}\right)$ e alumínio $\left(\mathrm{Al}_{\mathrm{o}}\right)$ oriundos de minerais de baixa cristalinidade foi obtido pela extração em solução ácida $(\mathrm{pH} 3,0)$ de oxalato de amônio no escuro (SCHWERTMANN, 1964). O teor de Fe $\left(\mathrm{Fe}_{\mathrm{h}}\right)$, considerado o teor total da fração argila, foi extraído por $\mathrm{HCl} 6 \mathrm{~mol} \mathrm{~L}^{-1}$ (DICK et al., 1988). A concentração de $\mathrm{Fe}$ e $\mathrm{Al}$ dos extratos foi quantificada em espectrofotômetro de absorção atômica.

Amostras da fração terra fina e de plintitas e petroplintitas de cada horizonte foram moídas e passadas em peneira de malha $0,05 \mathrm{~mm}$ para difração de raios $\mathrm{X}$ em pó. $\mathrm{O}$ equipamento utilizado foi um difratômetro de raios $\mathrm{X}$ operando com ânodo de cobre ( $\mathrm{Cu} \mathrm{K \alpha} 0,154 \mathrm{~nm})$ e filtro de níquel, velocidade de varredura de $1,2^{\circ} 2 \theta \mathrm{min}^{-1}$, faixa de varredura de $3^{\circ} \mathrm{a}$ $45^{\circ} 2 \theta$, com tensão de aceleração de $40 \mathrm{kV}$ e corrente de $40 \mathrm{~mA}$.

\section{RESULTADOS E DISCUSSÃO}

O relevo dos locais de estudo é semelhante, variando de plano a suave ondulado com solos derivados de rochas sedimentares da Formação Santa Maria. A classe textural dos solos, principalmente dos horizontes mais superficiais, é predominantemente arenosa, variando de franca a franco-arenosa (Tabela 1). Devido aos processos de eluviação e iluviação (FANNING \& FANNING, 1989), ocorre gradiente textural nos perfis em estudo, com acúmulo de argila no horizonte subsuperficial.

O P3 apresenta grande quantidade de material ferruginoso consolidado na forma de petroplintita, o que não ocorre nos perfis P1 e P2. Isso pode ser atribuído a um rebaixamento do lençol freático, condicionando melhor drenagem ao ambiente, que é confirmado pela presença de matiz mais avermelhado (2,5YR) em relação aos demais perfis, a partir de $128 \mathrm{~cm}$ da superfície (Tabela 1).

Em relação aos dados da tabela 1, observa-se que, nos horizontes $\mathrm{A}$ e $\mathrm{AB}$ do $\mathrm{P} 1$, temse ausência de Al trocável e valores de $\mathrm{pH}$ maiores que os demais perfis. Isso também reflete nos maiores valores saturação por bases na camada de $0-45 \mathrm{~cm}$, possivelmente, devido à correção da acidez promovida 
Tabela 1 - Profundidade dos horizontes, cor (úmida), classe textural e composição química dos perfis estudados.

\begin{tabular}{|c|c|c|c|c|c|c|c|c|c|}
\hline $\mathrm{Hz}$ & $\begin{array}{l}\text { Prof. } \\
\text { cm }\end{array}$ & $\begin{array}{l}\text { Cor } \\
\text { úmida }\end{array}$ & C. T. ${ }^{1}$ & $\begin{array}{c}\mathrm{pH} \\
\mathrm{H}_{2} \mathrm{O}\end{array}$ & $\mathrm{CTC}_{\mathrm{pH} 7}$ & $\mathrm{Al}^{+3}$ & $\mathrm{~V}$ & $\mathrm{~m}$ & $\mathrm{MO}^{2}$ \\
\hline \multicolumn{10}{|c|}{ 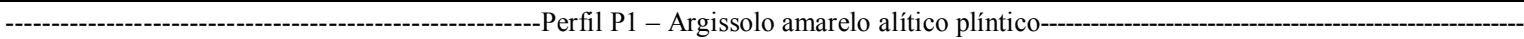 } \\
\hline Ap & $0-18$ & $10 \mathrm{YR} 3 / 2$ & $\mathrm{~F}^{3}$ & 6,7 & 7,8 & 0 & 82 & 0 & 2,2 \\
\hline A & $18-32$ & 10YR $3 / 2$ & $\mathrm{~F}$ & 6,7 & 14,5 & 0 & 82 & 0 & 2,2 \\
\hline $\mathrm{AB}$ & $32-45$ & $7,5 Y R 3 / 2$ & FA & 6,6 & 13,3 & 0 & 80 & 0 & 1,7 \\
\hline $\mathrm{BA}$ & $45-55$ & $10 \mathrm{YR} 3 / 3$ & FA & 6,8 & 11,4 & 0,4 & 65 & 5 & 1,5 \\
\hline Bt1 & $55-72$ & 7,5YR 4/6 & A & 5,2 & 11,6 & 3,7 & 31 & 51 & 1,4 \\
\hline $\mathrm{Bt} 2$ & $72-108$ & 7,5YR 4/2 & A & 5,2 & 6,3 & 4,6 & 17 & 81 & 1,0 \\
\hline $\mathrm{BC}$ & $108-120$ & $10 \mathrm{YR} 4 / 3$ & A & 5,2 & 6,4 & 5,0 & 22 & 78 & 0,6 \\
\hline $\mathrm{C}$ & $140+$ & $10 \mathrm{YR} 4 / 3$ & FA & 5,2 & 5,5 & 3,7 & 32 & 68 & 0,5 \\
\hline \multicolumn{10}{|c|}{-------Perfil P2 - Argissolo vermelho-amarelo Ta distrófico típico--- } \\
\hline A & $0-42$ & $10 \mathrm{YR} 4 / 2$ & $\mathrm{~F}$ & 5,2 & 27,0 & 4,0 & 35 & 30 & 2,5 \\
\hline $\mathrm{AB}$ & $42-69$ & $10 \mathrm{YR} 4 / 3$ & FA & 5,0 & 26,6 & 5,4 & 35 & 37 & 1,7 \\
\hline BA & $69-90$ & $7,5 \mathrm{YR} 4 / 4$ & A & 5,0 & 30,3 & 4,4 & 42 & 26 & 1,2 \\
\hline Bt1 & $90-111$ & $5 Y R 4 / 6$ & AS & 4,6 & 32,1 & 6,2 & 53 & 27 & 0,8 \\
\hline $\mathrm{Bt} 2$ & $111-135$ & $7,5 \mathrm{YR} 4 / 3$ & AS & 4,8 & 38,1 & 6,5 & 57 & 23 & 0,6 \\
\hline $\mathrm{BC}$ & $135+$ & $10 \mathrm{YR} 5 / 2$ & AS & 4,8 & 62,5 & 9,9 & 66 & 19 & 0,5 \\
\hline 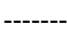 & . & 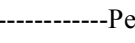 & Plinto & étrico & crecionário & sólico-- & - & 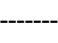 & ------- \\
\hline A1 & $0-43$ & $10 \mathrm{YR} 4 / 3$ & FA & 4,5 & 13,6 & 3,5 & 49 & 34 & 1,3 \\
\hline A2 & $43-64$ & $10 \mathrm{YR} 4 / 4$ & FA & 4,5 & 10,8 & 2,7 & 36 & 41 & 1,0 \\
\hline $\mathrm{E}$ & $64-97$ & $7,5 \mathrm{YR} 4 / 4$ & FA & 4,5 & 6,8 & 2,2 & 38 & 46 & 0,5 \\
\hline $\mathrm{Ec}$ & $97-109$ & 7,5YR 4/4 & FA & 4,7 & 5,3 & 1,6 & 51 & 37 & 0,5 \\
\hline $\mathrm{Bc} 1$ & $109-128$ & $5 \mathrm{YR} 4 / 4$ & $\mathrm{~F}$ & 4,7 & 9,6 & 2,5 & 54 & 32 & 0,3 \\
\hline $\mathrm{Bc} 2$ & $128-150$ & 2,5 YR $3 / 6$ & $\mathrm{~F}$ & 4,8 & 17,6 & 3,0 & 59 & 23 & 0,6 \\
\hline B1 & $150-186$ & $2,5 \mathrm{YR} 4 / 4$ & A & 4,7 & 20,8 & 3,9 & 51 & 27 & 0,6 \\
\hline B2 & $186-225$ & $2,5 \mathrm{YR} 4 / 4$ & FA & 4,7 & 15,8 & 4,0 & 43 & 37 & 0,3 \\
\hline $\mathrm{BC}$ & $225+$ & $2,5 \mathrm{YR} 4 / 6$ & FAA & 4,8 & 16,5 & 5,0 & 37 & 45 & 0,2 \\
\hline
\end{tabular}

${ }^{(1)}$ Classe textural. ${ }^{(2)}$ MO: Matéria orgânica. ${ }^{(3)}$ F: Franca. FA: Franco-argilosa. A: Argilosa. AS: Argilo-siltosa. FAA: Franco-argilo-arenosa.

pelo manejo da pastagem plantada. Já no P2 e P3, verifica-se uma situação típica de campo nativo, com acidez pronunciada, valores mais elevados de $\mathrm{Al}$ trocável e saturação por $\mathrm{Al}$ e baixa saturação por bases. A $\mathrm{CTC}_{\mathrm{pH}}$, normalmente é relacionada com o teor de matéria orgânica (MO), teor e tipo de argila. No P2, a presença de argila de atividade alta conferiu valores altos de $\mathrm{CTC}_{\mathrm{pH} 7}$, que variaram de 26,6 a $62,5 \mathrm{cmol}_{\mathrm{c}} \mathrm{kg}^{-1}$. Já nos demais perfis, possivelmente, a CTC tem maior relação com a interação entre teor de MO e de argila, do que com o tipo de minerais presentes nesta fração. Horizontes que têm presença de materiais ferruginosos (ANJOS et al., 2007) sofreram processo de ferrólise (ANDRADE et al., 1997) ou estão sujeitos à redução sazonal em períodos chuvosos (COELHO \& VIDAL-TORRADO, 2003), geralmente apresentam teor de Al trocável superior a $4 \mathrm{cmol}_{\mathrm{c}} \mathrm{kg}^{-1}$, associado à saturação por $\mathrm{Al}$ acima de $50 \%$ e saturação por bases inferior a $50 \%$. No entanto, embora a maioria dos horizontes associados a plintitas e/ou petroplintitas neste estudo apresentem $\mathrm{Al}$ trocável superior a $4 \mathrm{cmol}_{\mathrm{c}} \mathrm{kg}^{-1}$, somente o horizonte $\mathrm{BC}$ do $\mathrm{P} 1$ apresentou saturação por $\mathrm{Al}$ acima de 50\% e saturação por bases inferior a $50 \%$.

As concentrações de $\mathrm{Fe}$ (em todos os métodos de extração) no material de origem apresentam variações, apesar do material de origem desses solos ser o mesmo. Isso pode ser um indicativo de maior disponibilidade de Fe na solução do solo após o intemperismo e consequentemente formação de petroplitintas com maior concentração desse elemento no P1 (Tabela 2). Já para o P3, em que as concentrações de Fe nas petroplintitas são elevadas e o material de origem apresenta baixos valores, o rebaixamento do lençol freático e consequente drenagem fizeram com que o Fe migrasse do perfil, ora sendo perdido do sistema, ora sendo remobilizado na forma de concreções ferruginosas ou petroplintita,

Ciência Rural, v.43, n.6, jun, 2013. 
Tabela 2 - Teores de Fe e Al obtidos por diferentes extratores e suas respectivas relações.

\begin{tabular}{|c|c|c|c|c|c|c|c|c|}
\hline Horizonte & $\mathrm{Fe}_{\mathrm{h}}$ & ------ & $\begin{array}{l}\mathrm{Fe}_{\mathrm{o}} \\
\mathrm{kg}^{-1}\end{array}$ & $\mathrm{Al}_{\mathrm{d}}$ & $\mathrm{Al}_{\mathrm{o}}$ & $\mathrm{Fe}_{\mathrm{d}} / \mathrm{Fe}_{\mathrm{h}}$ & $\mathrm{Fe}_{\mathrm{o}} / \mathrm{Fe}_{\mathrm{d}}$ & $\mathrm{Al}_{\mathrm{o}} / \mathrm{Al}_{\mathrm{d}}$ \\
\hline \multicolumn{9}{|c|}{ 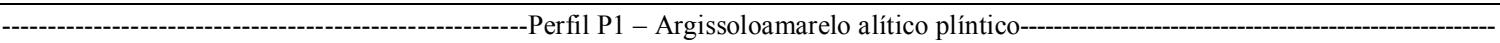 } \\
\hline Ap & 17,0 & 14,1 & 1,9 & 4,2 & 1,3 & 0,83 & 0,13 & 0,31 \\
\hline A & 17,9 & 15,7 & 2,0 & 6,0 & 0,8 & 0,88 & 0,13 & 0,13 \\
\hline $\mathrm{AB}$ & 27,9 & 17,0 & 2,0 & 6,8 & 1,7 & 0,61 & 0,12 & 0,25 \\
\hline $\mathrm{BA}$ & 23,0 & 18,8 & 1,6 & 4,0 & 1,6 & 0,82 & 0,09 & 0,40 \\
\hline $\mathrm{Bt} 1$ & 28,7 & 22,3 & 1,3 & 4,6 & 1,9 & 0,78 & 0,06 & 0,41 \\
\hline $\mathrm{Bt} 2$ & 32,1 & 23,4 & 0,6 & 3,8 & 1,6 & 0,73 & 0,03 & 0,42 \\
\hline $\mathrm{BC}-\mathrm{M}$ & 17,9 & 16,4 & 0,3 & 4,3 & 1,8 & 0,92 & 0,02 & 0,42 \\
\hline $\mathrm{BC}-\mathrm{N}$ & 42,9 & 34,3 & 0,7 & 2,4 & 1,8 & 0,80 & 0,02 & 0,75 \\
\hline $\mathrm{BC}-\mathrm{P}$ & 353,8 & 89,2 & 1,8 & 6,5 & 1,2 & 0,25 & 0,02 & 0,18 \\
\hline $\mathrm{C}-\mathrm{M}$ & 16,5 & 14,1 & 0,3 & 3,0 & 1,2 & 0,85 & 0,02 & 0,40 \\
\hline $\mathrm{C}-\mathrm{N}$ & 53,0 & 40,4 & 0,6 & 2,8 & 2,0 & 0,76 & 0,01 & 0,71 \\
\hline $\mathrm{C}-\mathrm{P}$ & 342,9 & 108,0 & 1,7 & 7,1 & 1,3 & 0,31 & 0,02 & 0,18 \\
\hline M.O. & 69,3 & 49,0 & 0,8 & 3,4 & 1,8 & 0,71 & 0,02 & 0,53 \\
\hline \multicolumn{9}{|c|}{ - } \\
\hline A & 12,5 & 12,0 & 2,3 & 4,1 & 1,4 & 0,96 & 0,19 & 0,34 \\
\hline $\mathrm{AB}$ & 16,4 & 13,8 & 1,9 & 4,4 & 1,5 & 0,84 & 0,14 & 0,34 \\
\hline $\mathrm{BA}$ & 31,2 & 19,8 & 1,3 & 6,7 & 1,6 & 0,63 & 0,07 & 0,24 \\
\hline Bt1 & 28,8 & 17,1 & 0,9 & 3,7 & 1,3 & 0,59 & 0,05 & 0,35 \\
\hline $\mathrm{Bt} 2-\mathrm{M}$ & 14,0 & 13,7 & 0,3 & 1,0 & 1,5 & 0,98 & 0,02 & 1,50 \\
\hline $\mathrm{Bt} 2-\mathrm{N}$ & 111,4 & 81,9 & 3,7 & 2,0 & 1,4 & 0,74 & 0,05 & 0,70 \\
\hline $\mathrm{BC}$ & 53,0 & 25,0 & 0,9 & 4,8 & 1,4 & 0,47 & 0,04 & 0,29 \\
\hline M.O ${ }^{1}$ & 23,4 & 21,8 & 0,6 & 1,3 & 1,2 & 0,93 & 0,03 & 0,92 \\
\hline $\mathrm{M} \mathrm{O}^{2}$ & 21,0 & 17,3 & 0,5 & 0,8 & 1,0 & 0,82 & 0,03 & 1,25 \\
\hline \multicolumn{9}{|c|}{ - } \\
\hline A1 & 8,5 & 8,0 & 0,5 & 0,7 & 0,6 & 0,94 & 0,06 & 0,86 \\
\hline $\mathrm{A} 2$ & 9,4 & 8,0 & 0,6 & 1,5 & 0,5 & 0,85 & 0,08 & 0,33 \\
\hline $\mathrm{E}$ & 14,0 & 6,7 & 0,3 & 0,9 & 0,4 & 0,48 & 0,04 & 0,44 \\
\hline $\mathrm{Ec}-\mathrm{M}$ & 20,3 & 5,5 & 0,1 & 0,5 & 0,2 & 0,27 & 0,02 & 0,40 \\
\hline$E c-P$ & 304,0 & 95,8 & 1,6 & 2,4 & 0,4 & 0,32 & 0,02 & 0,17 \\
\hline $\mathrm{Bc} 1-\mathrm{M}$ & 18,7 & 10,1 & 0,3 & 2,1 & 0,3 & 0,54 & 0,03 & 0,14 \\
\hline $\mathrm{Bc} 1-\mathrm{P}$ & 323,4 & 96,6 & 1,5 & 2,3 & 0,4 & 0,30 & 0,02 & 0,17 \\
\hline $\mathrm{Bc} 2-\mathrm{M}$ & 42,9 & 19,0 & 0,5 & 3,0 & 0,6 & 0,44 & 0,03 & 0,20 \\
\hline $\mathrm{Bc} 2-\mathrm{P}$ & 296,1 & 88,2 & 1,1 & 1,9 & 0,4 & 0,30 & 0,01 & 0,21 \\
\hline B1 & 31,2 & 22,5 & 0,4 & 2,8 & 0,8 & 0,72 & 0,02 & 0,29 \\
\hline B2 & 27,3 & 20,8 & 0,3 & 2,4 & 0,7 & 0,76 & 0,01 & 0,29 \\
\hline $\mathrm{BC}$ & 22,6 & 17,0 & 0,5 & 3,3 & 0,7 & 0,75 & 0,03 & 0,21 \\
\hline M.O. & 7,2 & 7,0 & 0,2 & 0,5 & 0,6 & 0,97 & 0,03 & 1,20 \\
\hline
\end{tabular}

M: Matriz do solo; N: plintita; P: petroplintita dos horizontes; M.O: material de origem. ${ }^{(1)}$ profundidade de $220-230 \mathrm{~cm} .{ }^{(2)}$ profundidade de $230 \mathrm{~cm}+$.

explicando os altos teores de Fe nas petroplintitas concomitante com os baixos teores no material de origem.

Os maiores teores de Fe foram extraídos com $\mathrm{HCl}\left(\mathrm{Fe}_{\mathrm{h}}\right)$. As amostras de petroplintitas apresentaram teores superiores, comparado com as demais. Os valores de $\mathrm{Fe}_{\mathrm{h}}$ variam de 7,2 a $353,8 \mathrm{~g} \mathrm{~kg}^{-1}$ (Tabela 2). Em todos os perfis, os valores aumentam em profundidade, sendo os valores mais elevados observados nas petroplintitas do horizonte $\mathrm{BC}$ e $\mathrm{C}$ do P1, semelhante ao encontrado por COELHO \& VIDAL-TORRADO (2003).

Os três perfis de solo possuem um acréscimo dos teores de $\mathrm{Fe}_{\mathrm{d}}$ em profundidade, revelando que a maior parte do Fe está na forma de óxidos de $\mathrm{Fe}$ pedogênicos. Nos horizontes em que não houve separação dos materiais ferruginosos, o 
maior teor de $\mathrm{Fe}_{\mathrm{d}}$ foi encontrado no horizonte $\mathrm{BC}$ do P2 $\left(25,0 \mathrm{~g} \mathrm{~kg}^{-1}\right)$ e o menor valor no horizonte Ec do P3 $\left(5,5 \mathrm{~g} \mathrm{~kg}^{-1}\right)$. Este último, por ser um horizonte em que os processos de perdas são intensos, associados à translocação e acumulação de materiais ricos em ferro em horizontes mais profundos ou ainda neste mesmo horizonte, a concentração de Fe ocorre na forma de petroplintitas. Nas amostras que sofreram separação, o maior conteúdo de $\mathrm{Fe}_{\mathrm{d}}$ foi encontrado nas petroplintitas do horizonte C do perfil P1 (108,0g $\mathrm{kg}^{-1}$ ). Em solos na Amazônia Ocidental, LIMA et al. (2006) também verificaram maiores teores de $\mathrm{Fe}_{\mathrm{d}}$ em Argissolos do que em Plintossolos e, comumente, os teores de $\mathrm{Fe}_{\mathrm{d}}$ nas petroplintitas são maiores (COELHO \& VIDAL-TORRADO, 2003) do que nas demais amostras ou horizontes, principalmente em se tratando de amostras de Argissolos.

Os menores valores de $\mathrm{Fe}_{\mathrm{d}}$ nos três perfis estudados são observados na matriz de solo, principalmente nos horizontes superficiais. Isso está relacionado à textura mais arenosa e ao maior conteúdo de matéria orgânica (SCHWERTMANN \& TAYLOR, 1989) nos horizontes superficiais que facilitam a perda do ferro e podem inibir a cristalização dos óxidos de ferro (LOVLEY, 1995), favorecendo, assim, a formação de compostos de baixa cristalinidade (SILVA NETO et al., 2008; ANJOS et al., 2007; DICK et al., 2005). O caso do P3 com os menores teores de $\mathrm{Fe}_{\mathrm{d}}$ e de matéria orgânica em relação ao P1 e P2, é um exemplo da ação conjunta da textura e matéria orgânica na translocação de coloides para a subsuperfície e inibição da cristalização dos óxidos de $\mathrm{Fe}$, respectivamente.

Os valores mais altos da concentração de $\mathrm{Fe}_{\mathrm{o}}$ foram encontrados nos horizontes $\mathrm{A}$ e $\mathrm{AB}$ $\left(2 \mathrm{~g} \mathrm{~kg}^{-1}\right)$ do P1 e no horizonte A $\left(2,3 \mathrm{~g} \mathrm{~kg}^{-1}\right)$ do P2 devido ao maior conteúdo de matéria orgânica nesses horizontes, que favorece a formação de óxidos de $\mathrm{Fe}$ de baixa cristalinidade. Já nas plintitas, o valor mais alto se encontra no horizonte B2-N $\left(3,7 \mathrm{~g} \mathrm{~kg}^{-1}\right)$ do P2. De acordo com COELHO \& VIDAL-TORRADO (2003), o aumento de óxidos de ferro de baixa cristalinidade $\left(\mathrm{Fe}_{0}\right)$ nas plintitas é proveniente da mobilização recente e deposição de $\mathrm{Fe}$ durante a formação das plintitas e que não tiveram tempo ou condições bioclimáticas favoráveis para a formação e cristalização de óxidos de $\mathrm{Fe}\left(\mathrm{Fe}_{\mathrm{d}}\right)$. Por outro lado, nas petroplintitas, os maiores valores são observados nos horizontes Ec-P $\left(1,6 \mathrm{~g} \mathrm{~kg}{ }^{-1}\right), \mathrm{Bc} 1-\mathrm{P}$ $\left(1,5 \mathrm{~g} \mathrm{~kg}^{-1}\right)$ do P3 e BC-P $\left(1,8 \mathrm{~g} \mathrm{~kg}^{-1}\right)$, C-P $\left(1,7 \mathrm{~g} \mathrm{~kg}^{-1}\right)$ do P1. Conforme COELHO \& VIDAL-TORRADO (2003), altos valores de $\mathrm{Fe}$ nas formas de baixa cristalinidade $\left(\mathrm{Fe}_{\mathrm{o}}\right)$ presentes em petroplintitas podem ser entendidos como um novo ciclo de formação de óxidos de ferro. Os autores acreditam que formas de Fe cristalinas (hematita e goethita) que se encontram no interior das petroplintitas podem ser dissolvidas e formar uma nova geração de óxidos de Fe de baixa cristalinidade, como, por exemplo, a ferrihidrita. Ainda ocorre a redução de $\mathrm{Fe}^{+3}$ para $\mathrm{Fe}^{2+}$ que pode percolar e acumular-se nos poros dos saprolitos e rochas (SCHWERTMANN \& KÄMPF, 1983) pela mobilidade que possuem no solo (BLUME \& SCHWERTMANN, 1969).

Os maiores teores de $\mathrm{Al}_{\mathrm{d}}$ encontram-se nos horizontes AB do P1 (6,8g kg-1) e BA do P2 (6,7g $\left.\mathrm{kg}^{-1}\right)$. Os teores de $\mathrm{Al}_{\mathrm{d}}$ podem ser considerados altos se comparados com os valores encontrados por LIMA et al. (2006), já que os teores variaram de 3,4 a 5,5g $\mathrm{kg}^{-1}$ para um Argissolo. Altos valores de $\mathrm{Al}_{\mathrm{d}}$ podem sugerir dissoluções de materiais aluminossilicatados de pequeno tamanho (FONTES \& WEED, 1991), além da presença de óxidos de Fe com substituição isomórfica por $\mathrm{Al}$ (CORREA et al., 2008). Para o $\mathrm{Al}_{\mathrm{o}}$ os maiores teores são encontrados nos horizontes B1 $\left(1,9 \mathrm{~g} \mathrm{~kg}^{-1}\right)$ e C-N $\left(2,0 \mathrm{~g} \mathrm{~kg}^{-1}\right)$ do P1. Observa-se que, no material de origem do $\mathrm{P} 2$, o teor de $\mathrm{Al}_{\mathrm{o}}$ é maior que o de $\mathrm{Al}_{\mathrm{d}}$, isso pode estar relacionado com o maior conteúdo de minerais aluminossilicatados de baixa cristalinidade (principalmente caulinita), tal como sugerido por McKEAGUE \& DAY (1966) e verificado nos difratogramas de raios $\mathrm{X}$ (Figura 1).

A relação $\mathrm{Fe}_{\mathrm{o}} / \mathrm{Fe}_{\mathrm{d}}$ variou de 0,01 a 0,13 , indicando o predomínio de óxidos de Fe cristalinos (INDA \& KÄMPF, 2003), o que é confirmado pelos difratogramas de raios $\mathrm{X}$ (Figura 1). Os maiores valores da relação $\mathrm{Fe}_{\mathrm{o}} / \mathrm{Fe}_{\mathrm{d}}$ são observados nos horizontes em que não há presença de plintita e petroplintita, ou seja, na matriz do solo. HUANG et al. (2008) encontram valores de $\mathrm{Fe}_{\mathrm{o}} / \mathrm{Fe}_{\mathrm{d}}$ mais elevados nas concreções, porém, os solos estudados por esses autores são da região subtropical da China e que apresentam altos teores de $\mathrm{Fe}_{\mathrm{o}}$, contrapondo-se aos deste estudo.

A matriz do solo apresentou composição mineralógica distinta das amostras de plintita e petroplintita analisadas por difratometria de raios $\mathrm{X}$ (Figura 1). Na matriz do solo de ambos os perfis, foram identificados reflexos intensos de caulinita e quartzo e reflexos de menor intensidade de esmectita e hematita, único óxido de Fe identificado. Nos difratogramas de raios $\mathrm{X}$ das plintitas do $\mathrm{P} 1$ (horizonte BC), os reflexos da hematita foram mais intensos que os da matriz do solo. Tais reflexos ainda aumentam a intensidade nas petroplintitas dos dois perfis, enquanto que os reflexos referentes à caulinita praticamente desaparecem. O maior teor de $\mathrm{Fe}_{\mathrm{d}}$ e a predominância de óxidos de $\mathrm{Fe}$ cristalinos $\left(\mathrm{Fe}_{\mathrm{o}} / \mathrm{Fe}_{\mathrm{d}}\right)$

Ciência Rural, v.43, n.6, jun, 2013. 


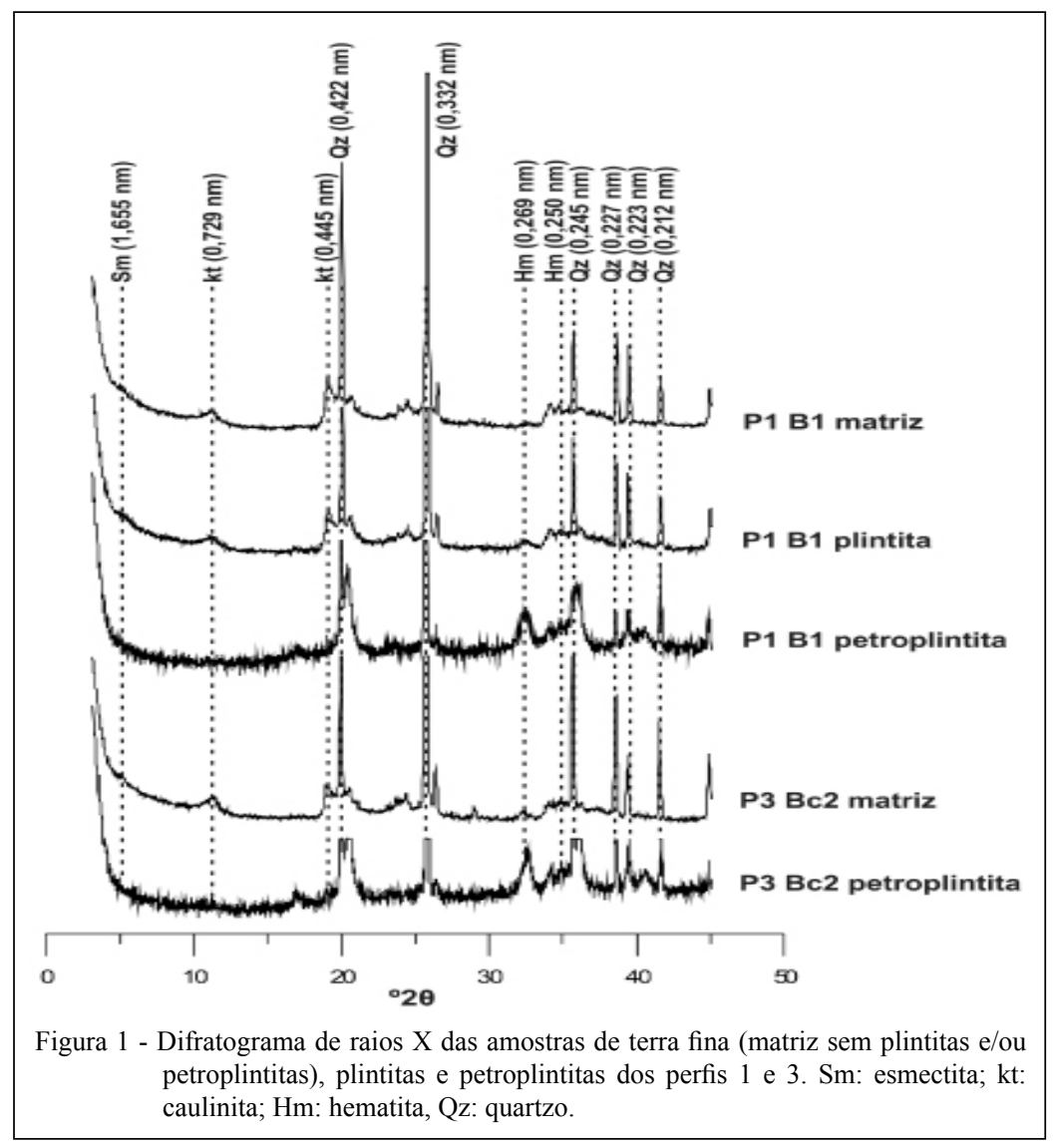

nas petroplintitas no $\mathrm{Bc} 2(\mathrm{P} 3)$ em relação à matriz do solo foi o que proporcionou os reflexos intensos referentes à hematita. Já no $\mathrm{P} 1$, a relação entre óxidos de Fe cristalinos e de baixa cristalinidade é semelhante para todas as amostras analisadas, sendo os reflexos afetados somente pelo teor de Fed.

\section{CONCLUSÃO}

Os horizontes dos solos apresentaram ampla variação de texturas e atributos químicos como $\mathrm{pH}, \mathrm{S}, \mathrm{V}, \mathrm{m}$ e $\mathrm{MO}$, nos quais essas variações estão associadas, principalmente, à ação antrópica. Processos de iluviação de compostos de ferro, dissoluções de materiais aluminossilicatados, dissolução de $\mathrm{Fe}_{\mathrm{d}}$ no interior das petroplintitas, indicam ser os principais processos pedogenéticos atuantes nos perfis, influenciando sobremaneira na morfologia, nos atributos químicos e mineralógicos das plintitas, petroplintitas e dos horizontes dos perfis. As concentrações de $\mathrm{Fe}_{\mathrm{h}}, \mathrm{Fe}_{\mathrm{d}}$ e $\mathrm{Fe}_{\mathrm{o}}$ seguiram a tendência: matriz do solo $<$ plintitas $<$ petroplintitas. A relação $\mathrm{Fe}_{\mathrm{o}} / \mathrm{Fe}_{\mathrm{d}}$ evidencia a predominância de óxidos Fe cristalinos nos perfis estudados. Dentre os óxidos de Fe, verificou-se predomínio do tipo hematita em todas as amostras avaliadas.

\section{AGRADECIMENTOS}

Os autores agradecem ao Conselho Nacional de Desenvolvimento Científico e Tecnológico (CNPq) pelo suporte financeiro e pela bolsa de produtividade em pesquisa do segundo autor.

\section{REFERÊNCIAS}

ANDRADE, H. et al. Pedogeomorfologia e micropedologia de uma seqüência Latossolo-Areia Quartzosa Hidromórfica sobre rochas cristalinas no Estado do Amazonas. Geonomos, v.5, n.1, p.5566, 1997. Disponível em: <http://www.igc.ufmg.br/geonomos/ PDFs/5_1_55_66_Andrade.pdf $>$. Acesso em: 25 mar. 2011.

ANJOS, L.H.C. et al. Caracterização e classificação de plintossolos no município de Pinheiro-MA. Revista Brasileira de Ciência Solo, v.31, n.5, p.1035-1044, 2007. Disponível em: <http://www.scielo.br/ scielo.php?script=sci arttext\&pid=S0100-06832007000500020>. Acesso em: 12 jan. 2010. doi: 10.1590/S0100-06832007000500020.

ANJOS, L.H.C. et al. Formation of soils with plinthite on a toposequence in Maranhão State, Brazil. Geoderma, v.64, n.3, p.257-279, 1995. Disponível em: <http://www.sciencedirect. com/science/article/pii/0016706194000223>. Acesso em: 24 mar. 2010. doi:10.1016/0016-7061(94)00022-3. 
BLUME, H.P.; SCHWERTMANN, U. Genetic evaluation of distribution of aluminum, iron, and manganese oxides. Soil Science Society of America. Proceedings, v.33, n.3, p.438444, 1969. Disponível em: <www.soils.org/publications/ sssaj/tocs/33/3>. Acesso em: 30 jun. 2010. doi: 10.2136/ sssaj1969.03615995003300030030.

COELHO, M.R.; VIDAL-TORRADO, P. Caracterização e gênese de perfis plínticos desenvolvidos de arenito do Grupo Bauru I. Química. Revista Brasileira de Ciência Solo, v.27, n.3, p.483-494, 2003. Disponível em: $<$ http://www.scielo.br/scielo.php?script=sci arttext\&pid=S0100-06832003000300011>. Acesso em: 29 mar. 2011. doi: $10.1590 / \mathrm{S} 0100-06832003000300011$.

CORRÊA, M.M. et al. Caracterização de óxidos de ferro de solos do ambiente tabuleiros costeiros. Revista Brasileira de Ciência do Solo, v.32, n.3, p.121-131, 2008. Disponível em: <http://www.scielo.br/scielo.php?pid=S0100$06832008000300011 \&$ script $=$ sci abstract\&tlng $=\mathrm{pt}>$. Acesso em 14 jul. 2011. doi: 10.1590/S0100-06832008000300011.

DICK, D.P. et al. Characteristics of soil organic matter of different Brazilian Ferralsols under native vegetation as function of soil depth. Geoderma, v.124, n.3/4, p.319-333, 2005. Disponivel em: <http://www.sciencedirect.com/science/article/pii/ S0016706104001259>. Acesso em: 23 ago. 2011. doi:10.1016/j. geoderma.2004.05.008.

DICK, D.P.; KÄMPF, N. Comparação da extração de ferro com HCL 6N, H2SO4 1:1 e ditionito-citrato-bicarbonato de sódio em horizontes B-latossólicos. Revista Brasileira de Ciência Solo, v.12, p.185-188, 1988.

EMPRESA BRASILEIRA DE PESQUISA AGROPECUÁRIA (EMBRAPA). Centro Nacional de Pesquisa de Solos. Manual de métodos de análise de solo. 2.ed. Rio de Janeiro, 1997. 212p.

EMPRESA BRASILEIRA DE PESQUISA AGROPECUÁRIA (EMBRAPA). Sistema brasileiro de classificação de solo. 2.ed. Brasília, 2006. 306p.

FANNING, D.S.; FANNING, M.C.B. Soil morphology, genesis and classification. New York: John Wiley \& Sons, 1989. 395p.

FONTES, M.P.F.; WEED, S.B. Iron oxides in selected brazilian oxisols: I. Mineralogy. Soil Science Society of America Journal, v.55, n.4, p.1143-1149, 1991. Disponível em: <www.soils.org/ publications/sssaj/tocs/55/4>. Acesso em: 12 abr. 2011. doi: 10.2136/sssaj1991.03615995005500040040.

HUANG, L. et al. Characteristics of micromorphology and element distribution of iron-manganese cutans in typical soils of subtropical China. Geoderma, v.146, n.1/2, p.40-47, 2008. Disponível em: <http:/www.sciencedirect.com/science/article/pii/ S0016706108001195>. Acesso em: 15 fev. 2011. doi: 10.1016/j. geoderma.2008.05.007.

INDA, A.V.; KÄMPF, N. Avaliação de procedimentos de extrações dos óxidos de ferro pedogênicos com ditionito-citrito-bicarbonato de sódio (DCB). Revista Brasileira de Ciência do Solo, v.27, n.6, p.1139-1147, 2003. Disponível em: <http://www.scielo.br/scielo. php?script $=$ sci_arttext\&pid $=$ S0100-06832003000600018\&lng $=$ p t\&nrm=iso\&tlng $=$ pt $>$. Acesso em: 10 jan. 2010 . doi: $10.1590 /$ S0100-06832003000600018.

LIMA, H.N. et al. Mineralogia e química de três solos de uma toposseqüência da bacia sedimentar do Alto Solimões, Amazônia Ocidental. Revista Brasileira de Ciência Solo, v.30, n.1, p.59-68, 2006. Disponível em: <http://www.scielo.br/scielo. php?script $=$ sci_arttext\&pid $=$ S0100-06832006000100007\&lng $=$ p t\&nrm=iso\&tlng=pt $>$. Acesso em: 10 fev. 2010. doi: 10.1590/ S0100-06832006000100007.

LOVLEY, D.R. Bioremediation of organic and metal contaminants with dissimilatory metal reduction. Journal Industrial Microbiology, v.14, n.2, p.85-93, 1995. Disponível em: <http:// www.geobacter.org/publication-files/7766214.pdf $>$. Acesso em: 20 fev. 2010. doi: 10.1007/BF01569889.

MALUF, J.R.T. Nova classificação climática do Estado do Rio Grande do Sul. Revista Brasileira de Agrometeorologia, v.8, n.1, p.141-150, 2000. Disponível em: <http://www.ufsm.br/rba/ p14181.html >. Acesso em: 25 fev. 2010.

McKEAGUE, J.A.; DAY, J.H. Dithionite-and oxalate-extractable $\mathrm{Fe}$ and $\mathrm{Al}$ as aids in differentiating various classes of soils. Canadian Journal of Soil Science, v.46, n.1, p.13-22, 1966. Disponível em: <http://pubs.aic.ca/doi/abs/10.4141/cjss66-003>. Acesso em: 25 mar. 2010. doi: 10.4141/cjss66-003.

MEHRA, O.P.; JACKSON, M.L. Iron oxide removal from soil and clays by dithionite-citrate system buffered with sodium bicarbonate. Clays and Clay Mineral, v.7, n.1, p.317-327, 1960. Disponível em: <http://www.clays.org/journal/archive/ volume\%207/7-1-317.pdf $>$. Acesso em: 25 jan. 2010 . doi: 10.1346/CCMN.1958.0070122.

NELSON, D.W.; SOMMERS, L.E. Total carbon, organic carbon and organic matter. In: SPARKS, D.L. et al. (Eds.). Methods of soil analysis: chemical methods. Part 3. Madison: Soil Science Society of America Journal, 1996. p.961-1010.

SANTOS, R.D. et al. Manual de descrição e coleta de solo no campo. 5.ed. Viçosa: SBCS, 2005. 100p.

SARTORI, P. Geologia e geomorfologia de Santa Maria. Ciência e Ambiente, v.39, p.19-42, 2009.

SCHWERTMANN, U.; KÄMPF, N. Óxidos de ferro jovens em ambientes pedogenéticos brasileiros. Revista Brasileira de Ciência Solo, v.7, n.3, p.251-255, 1983. Disponível em: <http:// cat.inist.fr/?aModele $=$ afficheN\&cpsidt $=9517524>$. Acesso em: 24 jan. 2010.

SCHWERTMANN, U.; TAYLOR, R.M. Iron oxides. In: DIXON, J.B.; WEED, S.B. (Eds.). Minerals in soil environments. 2.ed. Madison: Soil Science Society of America Journal, 1989. p.379-438.

SCHWERTMANN, U. Differenzierung der eisenoxide des bodens durch photochemische extraktion mit saurer ammoniumoxalate. Zeitschrift fuer Pflanzenernaehrung-Düngund Bodenkunde, v.105, n.5, p.94-202, 1964. Disponível em: <http://onlinelibrary. wiley.com/doi/10.1002/jpln.3591050303/abstract>. Acesso em: 20 fev. 2010. doi:10.1002/jpln.3591050303.

SILVA NETO, L. de F. da S. et al. Óxidos de ferro em latossolos tropicais e subtropicais brasileiros em plantio direto. Revista Brasileira de Ciência Solo, v.32, n.5, p.1873-1881, 2008. Disponível em: <http://www.scielo.br/pdf/rbcs/v32n5/08.pdf > . Acesso em: 25 mar. 2010. doi: 10.1590/S0100-06832008000500008.

STOLT, M.H. et al. Soil-landscape relationships in Virginia: I. Soil variability and parent material uniformity. Soil Science Society of America Journal, v.57, n.2, p.414-421, 1993. Disponível em: $<$ http://www.geo.msu.edu/schaetzl/871/Stolt\%20et\%20al2.pdf $>$. Acesso em: 25 fev. 2010.

STRECK, E.V. et al. Solos do Rio Grande do Sul. 2.ed. rev. e ampl. Porto Alegre: Emater/RS, 2008. 222p. 